Objectives: To assess AIDAI score and evaluate correlation between AIDAI and disease/response characteristics over 16 weeks (wks) of CAN treatment in CLUSTER.

Methods: CLUSTER study design and results have been presented. ${ }^{2}$ AIDAI was calculated as the sum of 12 items $^{1}$ for 30 consecutive days. AIDAI score was calculated if the first score was recorded $\geq 29$ days before baseline (BL). Missing items were imputed beyond last evaluable measurement by LOCF. Proportion of pts with inactive disease (ID; AIDAI score <9) was calculated at Wk 16. Correlation analysis of AIDAI with C-reactive protein (CRP), serum amyloid A (SAA), physician global assessment (PGA) and sheehan disability score (SDS), and child health questionnaire-psychological/physical (CHQ-PsCS/PCS) and short form 12-physical/mental (SF12-PCS/MCS) component summaries were performed at $\mathrm{BL}$ and Wk 16, with significance set at $\mathrm{p}<0.05$.

Results: Median AIDAI scores decreased over time (Fig 1). Proportion of pts with ID at Wk 16 was $52 \%$ in $\mathrm{crFMF}, 40 \%$ in HIDS/MKD and $46 \%$ in TRAPS cohorts. AIDAI at Wk 16 correlated significantly with: SDS in all 3 cohorts; PGA in HIDS/MKD and TRAPS; SF12-MCS in crFMF and HIDS/MKD (Table 1). CRP and SAA did not correlate with AIDAI.

Table 1. Correlation between AIDAI and disease activity/response variables at Week 16

\begin{tabular}{|c|c|c|c|}
\hline & \multicolumn{3}{|c|}{ Correlation coefficient $(95 \% \mathrm{Cl})$} \\
\hline & $\operatorname{crFMF}(\mathrm{N}=63)$ & HIDS/MKD (N=72) & TRAPS $(\mathrm{N}=46)$ \\
\hline CRP & $-0.12(-0.36 ; 0.14)$ & $0.23(-0.01 ; 0.45)$ & $0.12(-0.19 ; 0.42)$ \\
\hline SAA & $-0.01(-0.27 ; 0.25)$ & $-0.05(-0.30 ; 0.21)$ & $0.06(-0.26 ; 0.37)$ \\
\hline PGA & $0.23(-0.02 ; 0.46)$ & $0.35^{\S}(0.12 ; 0.55)$ & $0.73^{\star}(-0.54 ; 0.85)$ \\
\hline $\mathrm{CHQ}-\mathrm{PsCS}$ & $-0.18(-0.56 ; 0.26)$ & $-0.25(-0.55 ; 0.11)$ & $-0.33(-0.72 ; 0.22)$ \\
\hline $\mathrm{CHQ}-\mathrm{PCS}$ & $-0.33(-0.66 ; 0.11)$ & $-0.46^{\S}(-0.70 ;-0.14)$ & $-0.48(-0.80 ; 0.04)$ \\
\hline SF12-PCS & $-0.26(-0.57 ; 0.11)$ & $-0.23(-0.68 ; 0.35)$ & $-0.52^{\ddagger}(-0.81 ;-0.03)$ \\
\hline SF12-MCS & $-0.45^{\ddagger}(-0.70 ;-0.10)$ & $-0.55^{\ddagger}(-0.84 ;-0.03)$ & $0.09(-0.43 ; 0.56)$ \\
\hline SDS & $0.47^{\dagger}(0.22 ; 0.67)$ & $0.37^{\S}(0.10 ; 0.59)$ & $0.41^{\ddagger}(0.06 ; 0.67)$ \\
\hline
\end{tabular}

${ }^{*} p<0.0001 ;{ }^{\dagger} p<0.001 ;{ }^{\S} p<0.01 ;{ }^{\ddagger} p<0.05$.

Fig 1. Median AIDAI scores over 16 weeks

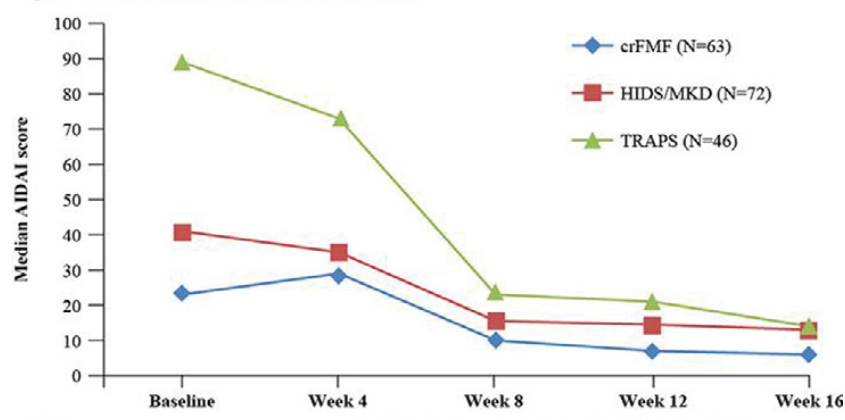

AIDAI score was calculated as the sum of all 12 daily items ( $\mathrm{YES}=1$ or $\mathrm{NO}=0$ ) during 30 days. Missing AIDAI assessments between first and last AIDAI assessments were imputed as NO. Missing AIDAI assessments between first and last AIDAI assessments were in

LOCF. last observation carried forward.

Conclusions: CAN demonstrated rapid and sustained disease control assessed with AIDAI over 16 wks. Approximately half of crFMF and TRAPS pts, and $40 \%$ of HIDS/MKD patients had inactive disease after 16 wks of treatment. AIDAI improvements at Wk 16 correlated with patient and physician driven evaluations (PGA, SF12-MCS and SDS). CRP and SAA are indicators of response to treatment, rather than a disease activity parameter.

References:

[1] Piram M, et al. Ann Rheum Dis. 2014;73: 2168-73.

[2] De Benedetti F, et al. Ann Rheum Dis. 2016;75:615-6.

Disclosure of Interest: I. Kone-Paut Grant/research support from: Novartis, $\mathrm{SOBI}$ and Roche, Consultant for: Novartis, SOBI, Pfizer, AbbVie and Roche, M. Piram Consultant for: Novartis, Pfizer, AbbVie, Speakers bureau: Novartis, S. Benseler Consultant for: Novartis, SOBI and AbbVie, M. Hofer Consultant for: Novartis and AbbVie, H. Lachmann Consultant for: Novartis, SOBI, Takeda and GSK, Speakers bureau: Novartis and SOBI, H. Hoffman Consultant for: Novartis, Speakers bureau: Novartis, M. Gattorno Grant/research support from: Novartis and SOBI, Consultant for: Novartis and SOBI, J. Frenkel Grant/research support from: Novartis and SOBI, J. Kuemmerle-Deschner Grant/research support from: Novartis, Consultant for: Novartis, SOBI and Baxalta, S. Ozen Speakers bureau: Novartis and SOBI, J. Levy Paid instructor for: Novartis, C. Karyekar Employee of: Novartis, F. De Benedetti Grant/research support from: Pfizer, AbbVie, Roche,

Novartis, Novimmune and BMS

DOI: 10.1136/annrheumdis-2017-eular.5655

\section{THU0510 UKRAINIAN REGISTRY OF JIA PATIENTS RECEIVING BIOLOGICS: IMPLEMENTATION INTO CLINICAL PRACTICE AND RECENT DATA}

I.V. Biliavska ${ }^{1}$, V.M. Kovalenko ${ }^{1}$, O.P. Bortkevych ${ }^{1}$, O.O. Garmish ${ }^{1}$, Y.E. Boyko ${ }^{2}$, L.I. Omelchenko $^{1}$, O.A. Oshlyanska ${ }^{1}$, T.V. Marushko ${ }^{1}$, L.F. Bogmat ${ }^{3}$,

N.S. Shevchenko ${ }^{3}$. 1 Task Force, Ukrainian association of Rheumatologists, Kyiv;
${ }^{2}$ Task Force, Ukrainian association of Rheumatologists, Lviv; ${ }^{3}$ Task Force, Ukrainian association of Rheumatologists, Kharkiv, Ukraine

Background: The situation with a treatment of juvenile idiopathic arthritis (JIA) is complicated in Ukraine. Mainly due to financial reason biological therapy is hardly accessible for Ukrainian patients. Nevertheless, some patients with JIA receive biologics within governmental programme. Clinical data of these patients were scattered and not systematized until 2014. The Task Force of Ukrainian Association of rheumatologists, by analogy to other European registries, has developed a registry of JIA patients receiving biologics. Ukrainian register of JIA patients is an observational, prospective, non-interventional clinical study.

Objectives: The main aim of Ukrainian register of JIA patients is an assessment of long-term safety, efficacy and cost of biological treatment of JIA. in the routine clinical practice.

Methods: 33 clinical sites from different regions of Ukraine are participating. Inclusion criteria: i) diagnosis of JIA: ii)age $\leq 2$ years old, iii)planned start of biological therapy due to JIA; iv)negative screening for tuberculosis; v)provided informed consent. Patients have undergone standard clinical assessment every 3 months. Disease activity is measured using JADAS27 in all age groups. ANA, HLAB27, RF and anti-CCP detection are highly recommended. Uveitis and other comorbid conditions should also be fixed.

Results: 339 patients were enrolled into the study during 3 years. $64 \%$ of patients are girls. Mean age-10.98 \pm 4.41 years, with mean disease duration $-5.81 \pm 3.48$ years. The duration of the period between diagnosis and biologics start was $54.31 \pm 40.28$ months. Comorbid conditions were found in $41.03 \%$ patients. In $14.65 \%$ of the patients uveitis was diagnosed. Most common JIA subtypes in patients receiving biologics are pJIA with negative RF $(45 \%)$, sJIA (20\%), enthesitis-associated JIA (11\%) and persistent oligoarthritis (11\%). 67.3\% of enrolled patients received adalimumab (ADA); $27.9 \%>$ tocilizumab (TOZ) and $4.8 \%$ > etanercept, respectively. During observational period biologics was discontinued in $19.8 \%$ of patients due to different reasons: adverse events were observed in $6.7 \%$ (ADA) and $16.7 \%$ (TOZ), insufficient efficiency of $23.3 \%$ (ADA) and 33.3\% (TOZ), remission - 6.7\% (ADA); drug absence - 63.3\% (ADA) and $50 \%$ (TOZ), respectively. Comparative analysis of ADA and TOZ efficacy was performed in the 144 patients with pJIA with negative RF. Administration either ADA or TOZ resulted in statistically significant reducing of disease activity according to JADAS27. In ADA group after 3 months of administration JADAS27 decreased from $16.3 \pm 10.3$ to $10.0 \pm 7.8(p<0.00001)$. In TOZ group after 3 months of administration JADAS27 reduced from $22.2 \pm 12.2$ to $13.1 \pm 9.1 \quad(p=0.0012)$. The functional disability of the patients also statistically significant decrease in both treatment group starting from 3 months of administration: $1.1 \pm 0.8$ to $0.7 \pm 0.7$ $(p=0.0081)$ in ADA and $1.6 \pm 1.0$ to $1.0 \pm 0.7$ in TOZ, respectively.

Conclusions: Ukrainian national registry of JIA patients provides real-life longterm data concerning safety, efficacy, outcomes and comparative analysis of biologics in Ukrainian population of JIA patients. Data collection continues and the data received expected to be a background for clinical decision-making in future.

Disclosure of Interest: None declared

DOI: 10.1136/annrheumdis-2017-eular.4681

\section{THU0511 TOCILIZUMAB IS A PROMISING TREATMENT OPTION FOR THERAPY RESISTENT JUVENILE LOCALISED SCLERODERMA PATIENTS}

I. Foeldvari ${ }^{1}$, J. Anton ${ }^{2}$, M. Friswell ${ }^{3}$, B. Bica ${ }^{4}$, J. de Inocencio ${ }^{5}$, A. Aquilani ${ }^{6}$, N. Helmus ${ }^{1} .{ }^{1}$ Hamburg Center for Pediatric and Adolescent Rheumatology, Am Schön Klinik Eilbek, Hamburg, Germany; ${ }^{2}$ Sant Joan de Déu Hospital,

Barcelona, Spain; ${ }^{3}$ Great North Children's Hospital, Newcastle, United Kingdom; ${ }^{4}$ Universidade Federal do Rio de Janeiro, Rio de Janeiro, Brazil; ${ }^{5}$ Pediatric Rheumatology Unit University Hospital 12 de Octubre, Madrid, Spain; ${ }^{6}$ Ospedale Bambino Gesù, Rome, Italy

Background: Juvenile localised scleroderma (j|Sc) is an orphan disease. Most patient respond to treatment ot methotrexate or mycophenolate. In case of nonresponse or partial response, based on the promising tocilizumab (TOC) data of adult systemic sclerosis studies, TOC seems to be a promising option. There is no publication regarding the effectiveness of tocilizumab in jLS.

Objectives: To assess the effectivity of TOC in jISC patients, who had nonresponse or partial response on conventional therapy.

Methods: Participants of the pediatric rheumatology email board were asked, if they follow patients with jISc, who are treated with TOC. Clinical characteristics and the response to TOC was assessed.

Results: Six centers responded to the survey from the email board, with around 800 participatns, and reported 11 patients. The mean age of the patients at disease onset was 5.5 years. Disease duration at time of the intiation of TOC was 53.5. months (range 9 to 109). 5 patients had linear subtype, 3 of them with facial involvement, 2 of them Parry Romberg and one of them coup de sabre. Three had generalized subtype, 2 mixed subtype and 1 limited subtype/morphea. Before starting TOC patients received 10/11 Methotrexate, 7/11 Mycophenolate, 1 abatacept and 1 anti-TNF therapy. Reason to start TOC was in 9 patients increase in Localised Scleroderma Activity Index [1] (mLoSSI). In two patients increased extracutaneous activity was the indication, in one increased activity of arthritis and in the other increased activity of the central nervous system involvement. 